\title{
Gunrock: A High-Performance Graph Processing Library on the GPU
}

\author{
Yangzihao Wang, Andrew Davidson*, Yuechao Pan, Yuduo $\mathrm{Wu}^{\dagger}$, Andy Riffel, John D. Owens \\ University of California, Davis \\ \{yzhwang, aaldavidson, ychpan, yudwu, atriffel, jowens $\}$ @ucdavis.edu
}

\begin{abstract}
For large-scale graph analytics on the GPU, the irregularity of data access/control flow and the complexity of programming GPUs have been two significant challenges for developing a programmable high-performance graph library. "Gunrock," our high-level bulksynchronous graph-processing system targeting the GPU, takes a new approach to abstracting GPU graph analytics: rather than designing an abstraction around computation, Gunrock instead implements a novel data-centric abstraction centered on operations on a vertex or edge frontier. Gunrock achieves a balance between performance and expressiveness by coupling high-performance GPU computing primitives and optimization strategies with a highlevel programming model that allows programmers to quickly develop new graph primitives with small code size and minimal GPU programming knowledge. We evaluate Gunrock on five graph primitives (BFS, BC, SSSP, CC, and PageRank) and show that Gunrock has on average at least an order of magnitude speedup over Boost and PowerGraph, comparable performance to the fastest GPU hardwired primitives, and better performance than any other GPU high-level graph library.
\end{abstract}

\section{Introduction}

Graphs are ubiquitous data structures that can represent relationships between people (social networks), computers (the Internet), biological and genetic interactions, and elements in unstructured meshes, just to name a few. In this paper, we describe "Gunrock," our graphics processor (GPU)-based system for graph processing that delivers high performance in computing graph analytics with its high-level, data-centric parallel programming model. Unlike previous GPU graph programming models that focus on sequencing computation steps, our data-centric model's key abstraction is the frontier, a subset of the edges or vertices within the graph that is currently of interest. All Gunrock operations are bulk-synchronous and manipulate this frontier, either by computing on values within it or by computing a new frontier from it.

At a high level, Gunrock targets graph primitives that are iterative, convergent processes. Among the graph primitives we have

\footnotetext{
${ }^{*}$ Currently an employee at Google.
}

${ }^{\dagger}$ Currently an employee at IBM.

Permission to make digital or hard copies of part or all of this work for personal or classroom use is granted without fee provided that copies are not made or distributed for profit or commercial advantage and that copies bear this notice and the full citation on the first page. Copyrights for components of this work owned by others than ACM must be honored. Abstracting with credit is permitted. To copy otherwise, to republish, to post on servers, or to redistribute to lists, contact the Owner/Author. Request permissions from permissions@acm.org or Publications Dept., ACM, Inc., fax +1 (212) 869-0481. Copyright 2016 held by Owner/Author. Publication Rights Licensed to ACM.

PPoPP '16 March 12-16, 2016, Barcelona, Spain

Copyright (c) 2016 ACM 978-1-4503-4092-2/16/03, \$15.00

DOI: http://dx.doi.org/10.1145/2851141.2851145 implemented and evaluated in Gunrock, we focus in this paper on breadth-first search (BFS), single-source shortest path (SSSP), betweenness centrality (BC), PageRank, and connected components (CC). Though the GPU's excellent peak throughput and energy efficiency [17] have been demonstrated across many application domains, these applications often exploit regular, structured parallelism. The inherent irregularity of graph data structures leads to irregularity in data access and control flow, making an efficient implementation on GPUs a significant challenge.

Our goal with Gunrock is to deliver the performance of customized, complex GPU hardwired graph primitives with a highlevel programming model that allows programmers to quickly develop new graph primitives. To do so, we must address the chief challenge in a highly parallel graph processing system: managing irregularity in work distribution. Gunrock integrates sophisticated load-balancing and work-efficiency strategies into its core. These strategies are hidden from the programmer; the programmer instead expresses what operations should be performed on the frontier rather than how those operations should be performed. Programmers can assemble complex and high-performance graph primitives from operations that manipulate the frontier (the "what") without knowing the internals of the operations (the "how").

Our contributions are as follows:

1. We present a novel data-centric abstraction for graph operations that allows programmers to develop graph primitives at a high level of abstraction while simultaneously delivering high performance. This abstraction, unlike the abstractions of previous GPU programmable frameworks, is able to elegantly incorporate profitable optimizations-kernel fusion, push-pull traversal, idempotent traversal, and priority queues-into the core of its implementation.

2. We design and implement a set of simple and flexible APIs that can express a wide range of graph processing primitives at a high level of abstraction (at least as simple, if not more so, than other programmable GPU frameworks).

3. We describe several GPU-specific optimization strategies for memory efficiency, load balancing, and workload management that together achieve high performance. All of our graph primitives achieve comparable performance to their hardwired counterparts and significantly outperform previous programmable GPU abstractions.

4. We provide a detailed experimental evaluation of our graph primitives with performance comparisons to several CPU and GPU implementations.

Gunrock is currently available in an open-source repository at http://gunrock.github.io/ and is currently available for use by external developers. 


\section{Related Work}

This section discusses the research landscape of large-scale graph analytics frameworks in four fields:

1. Single-node CPU-based systems, which are in common use for graph analytics today, but whose serial or coarse-grained-parallel programming models are poorly suited for a massively parallel processor like the GPU;

2. Distributed CPU-based systems, which offer scalability advantages over single-node systems but incur substantial communication cost, and whose programming models are also poorly suited to GPUs;

3. GPU "hardwired," low-level implementations of specific graph primitives, which provide a proof of concept that GPU-based graph analytics can deliver best-in-class performance. However, best-of-class hardwired primitives are challenging to even the most skilled programmers, and their implementations do not generalize well to a variety of graph primitives; and

4. High-level GPU programming models for graph analytics, which often recapitulate CPU programming models (e.g., CuSha and MapGraph use PowerGraph's GAS programming model, Medusa uses Pregel's messaging model). The best of these systems incorporate generalized load balance strategies and optimized GPU primitives, but they generally do not compare favorably in performance with hardwired primitives due to the overheads inherent in a high-level framework and the lack of primitive-specific optimizations.

\subsection{Single-node and Distributed CPU-based Systems}

Parallel graph analytics frameworks provide high-level, programmable, high-performance abstractions. The Boost Graph Library (BGL) is among the first efforts towards this goal, though its serial formulation and $\mathrm{C}++$ focus together make it poorly suited for a massively parallel architecture like a GPU. Designed using the generic programming paradigm, the parallel BGL [13] separates the implementation of parallel algorithms from the underlying data structures and communication mechanisms. While many BGL implementations are specialized per algorithm, its breadth_first_visit pattern (for instance) allows sharing common operators between different graph algorithms. Pregel [20] is Google's effort at large-scale graph computing. It follows the Bulk Synchronous Parallel (BSP) model. A typical application in Pregel is an iterative convergent process consisting of global synchronization barriers called super-steps. The computation in Pregel is vertex-centric and based on message passing. Its programming model is good for scalability and fault tolerance. However, in standard graph algorithms in most Pregel-like graph processing systems, slow convergence arises from graphs with structure. GraphLab [19] allows asynchronous computation and dynamic asynchronous scheduling. By eliminating message-passing, its programming model isolates the user-defined algorithm from the movement of data, and therefore is more consistently expressive. PowerGraph [12] uses the more flexible Gather-Apply-Scatter (GAS) abstraction for power-law graphs. It supports both BSP and asynchronous execution. For the load imbalance problem, it uses vertex-cut to split high-degree vertices into equal degree-sized redundant vertices. This exposes greater parallelism in natural graphs Ligra [32] is a CPU-based graph processing framework for shared memory. It uses a similar operator abstraction for doing graph traversal. Its lightweight implementation is targeted at shared memory architectures and uses CilkPlus for its multithreading implementation. Galois $[26,28]$ is a graph system for shared memory based on a different operator abstraction that supports priority scheduling and dynamic graphs and processes on subsets of vertices called active elements. However, their model does not abstract the internal details of the loop from the user. Users have to generate the active elements set directly for different graph algorithms. Help is a library that provides high-level primitives for large-scale graph processing [29]. Using the primitives in Help is more intuitive and much faster than using the APIs of existing distributed systems. Green-Marl [15] is a domain-specific language for writing graph analysis algorithms on shared memory with built-in breadth-first search (BFS) and depth-first search (DFS) primitives in its compiler. Its language approach provides graph-specific optimizations and hides complexity. However, the language does not support operations on arbitrary sets of vertices for each iteration, which makes it difficult to use for traversal algorithms that cannot be expressed using a BFS or DFS.

\subsection{Specialized Parallel Graph Algorithms}

Recent work has developed numerous best-of-breed, hardwired implementations of many graph primitives. Merrill et al. [24]'s linear parallelization of the BFS algorithm on the GPU had significant influence in the field. They proposed an adaptive strategy for loadbalancing parallel work by expanding one node's neighbor list to one thread, one warp, or a whole block of threads. With this strategy and a memory-access efficient data representation, their implementation achieves high throughput on large scale-free graphs. Beamer et al.'s recent work on a very fast BFS for shared memory machines [1] uses a hybrid BFS that switches between top-down and bottomup neighbor-list-visiting algorithms according to the size of the frontier to save redundant edge visits. The current fastest connectedcomponent algorithm on the GPU is Soman et al.'s work [34] based on two PRAM connected-component algorithms [14]. There are several parallel Betweenness Centrality implementations on the GPU $[10,22,27,31]$ based on the work from Brandes and Ulrik [2]. Davidson et al. [5] proposed a work-efficient SingleSource Shortest Path algorithm on the GPU that explores a variety of parallel load-balanced graph traversal and work organization strategies to outperform other parallel methods. After we discuss the Gunrock abstraction in Section 3.1, we will discuss these existing hardwired GPU graph algorithm implementations using Gunrock terminology.

\subsection{High-level GPU Programming Models}

In Medusa [37], Zhong and He presented their pioneering work on a high-level GPU-based system for parallel graph processing, using a message-passing model. CuSha [18], targeting a GAS abstraction, implements the parallel-sliding-window (PSW) graph representation on the GPU to avoid non-coalesced memory access. CuSha additionally addresses irregular memory access by preprocessing the graph data structure ("G-Shards"). Both frameworks offer a small set of user-defined APIs but are challenged by load imbalance and thus fail to achieve the same level of performance as low-level GPU graph implementations. MapGraph [8] also adopts the GAS abstraction and achieves some of the best performance results for programmable single-node GPU graph computation.

\section{The Gunrock Abstraction and Implementation}

\subsection{Gunrock's Abstraction}

Gunrock targets graph operations that can be expressed as iterative convergent processes. By "iterative," we mean operations that may require running a series of steps repeatedly; by "convergent," we mean that these iterations allow us to approach the correct answer and terminate when that answer is reached. This target is similar to most high-level graph frameworks.

Where Gunrock differs from other frameworks, particularly other GPU-based frameworks, is in our abstraction. Rather than focusing on sequencing steps of computation, we instead focus on manipulating a data structure, the frontier of vertices or edges 
that represents the subset of the graph that is actively participating in the computation. It is accurate to say that for many (but not all) computations, the sequence of operations that result from our abstraction may be similar to what another abstraction may produce. Nonetheless, we feel that thinking about graph processing in terms of manipulations of frontier data structures is the right abstraction for the GPU. We support this thesis qualitatively in this section and quantitatively in Section 5.

One important consequence of designing our abstraction with a data-centered focus is that Gunrock, from its very beginning, has supported both vertex and edge frontiers, and can easily switch between them within the same graph primitive. We can, for instance, generate a new frontier of neighboring edges from an existing frontier of vertices. In contrast, gather-apply-scatter (PowerGraph/GAS) and message-passing (Pregel) abstractions are focused on operations on vertices and cannot easily support edge frontiers within their abstractions.

In our abstraction, we expose bulk-synchronous "steps" that manipulate the frontier, and programmers build graph primitives from a sequence of steps. Different steps may have dependencies between them, but individual operations within a step can be processed in parallel. For instance, a computation on each vertex within the frontier can be parallelized across vertices, and updating the frontier by identifying all the vertices neighboring the current frontier can also be parallelized across vertices. BSP operations are well-suited for efficient implementation on the GPU because they exhibit enough parallelism to keep the GPU busy and do not require expensive fine-grained synchronization or locking operations.

The graph primitives we describe in this paper use three Gunrock steps - advance, filter, and compute — each of which manipulate the frontier in a different way (Figure 1).

Advance An advance step generates a new frontier from the current frontier by visiting the neighbors of the current frontier. A frontier can consist of either vertices or edges, and an advance step can input and output either kind of frontier. Advance is an irregularly-parallel operation for two reasons: (1) different vertices in a graph have different numbers of neighbors and (2) vertices share neighbors, so an efficient advance is the most significant challenge of a GPU implementation.

The generality of Gunrock's advance allows us to use the same advance implementation across a wide variety of interesting graph operations. For instance, we can utilize Gunrock advance operators to: 1) visit each element in the current frontier while updating local values and/or accumulating global values (e.g., BFS distance updates); 2) visit the vertex or edge neighbors of all the elements in the current frontier while updating source vertex, destination vertex, and/or edge values (e.g., distance updates in SSSP); 3) generate edge frontiers from vertex frontiers or vice versa (e.g., BFS, SSSP, depth-first search, etc.); or 4) pull values from all vertices 2 hops away by starting from an edge frontier, visiting all the neighbor edges, and returning the far-end vertices of these neighbor edges. As a result, we can concentrate our effort on solving one problem (implementing an efficient advance) and see that effort reflected in better performance on other traversal-based graph operations.

Filter A filter step generates a new frontier from the current frontier by choosing a subset of the current frontier based on programmerspecified criteria. Though filtering is an irregular operation, using parallel scan for efficient filtering is well-understood on GPUs. Gunrock's filters can either 1) split vertices or edges based on a filter (e.g., SSSP's delta-stepping), or 2) compact out filtered items to throw them away (e.g., duplicate vertices in BFS, SSSP, and BC).

Compute A programmer-specified compute step defines an operation on all elements (vertices or edges) in the current frontier;
Gunrock then performs that operation in parallel across all elements. Because this parallelism is regular, computation is straightforward to parallelize in a GPU implementation. Many simple graph primitives (e.g., computing the degree distribution of a graph) can be expressed as a single computation step.
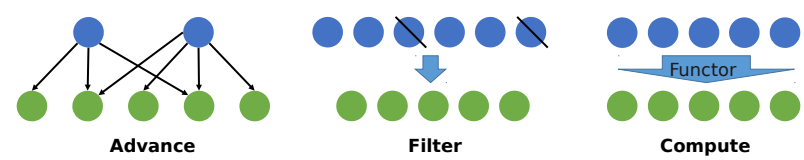

Figure 1: Three operators in Gunrock's data-centric abstraction convert a current frontier (in blue) into a new frontier (in green).

Gunrock primitives are assembled from a sequence of these steps, which are executed sequentially: one step completes all of its operations before the next step begins. Typically, Gunrock graph primitives run to convergence, which on Gunrock usually equates to an empty frontier; as individual elements in the current frontier reach convergence, they can be filtered out of the frontier. Programmers can also use other convergence criteria such as a maximum number of iterations or volatile flag values that can be set in a computation step.

Expressing SSSP in programmable GPU frameworks SSSP is a reasonably complex graph primitive that computes the shortest path from a single node in a graph to every other node in the graph. We assume weights between nodes are all non-negative, which permits the use of Dijkstra's algorithm and its parallel variants. Efficiently implementing SSSP continues to be an interesting problem in the GPU world $[3,5,6]$.

The iteration starts with an input frontier of active vertices (or a single vertex) initialized to a distance of zero. First, SSSP enumerates the sizes of the frontier's neighbor list of edges and computes the length of the output frontier. Because the neighbor edges are unequally distributed among the frontier's vertices, SSSP next redistributes the workload across parallel threads. This can be expressed within an advance frontier. In the final step of the advance frontier, each edge adds its weight to the distance value at its source value and, if appropriate, updates the distance value of its destination vertex. Finally, SSSP removes redundant vertex IDs (specific filter), decides which updated vertices are valid in the new frontier, and computes the new frontier for the next iteration.

Algorithm 1 provides more detail of how this algorithm maps to Gunrock's abstraction.

Gunrock maps one SSSP iteration onto three Gunrock steps: (1) advance, which computes the list of edges connected to the current vertex frontier and (transparently) load-balances their execution; (2) compute, to update neighboring vertices with new distances; and (3) filter, to generate the final output frontier by removing redundant nodes, optionally using a 2-level priority queue, whose use enables delta-stepping (a binning strategy to reduce overall workload [5, 25]). With this mapping in place, the traversal and computation of path distances is simple and intuitively described, and Gunrock is able to create an efficient implementation that fully utilizes the GPU's computing resources in a load-balanced way.

\subsection{Alternative Abstractions}

In this section we discuss several alternative abstractions designed for graph processing on various architectures.

Gather-apply-scatter (GAS) abstraction The GAS abstraction was first applied on distributed systems [12]. PowerGraph's vertexcut splits large neighbor lists, duplicates node information, and deploys each partial neighbor list to different machines. Working 


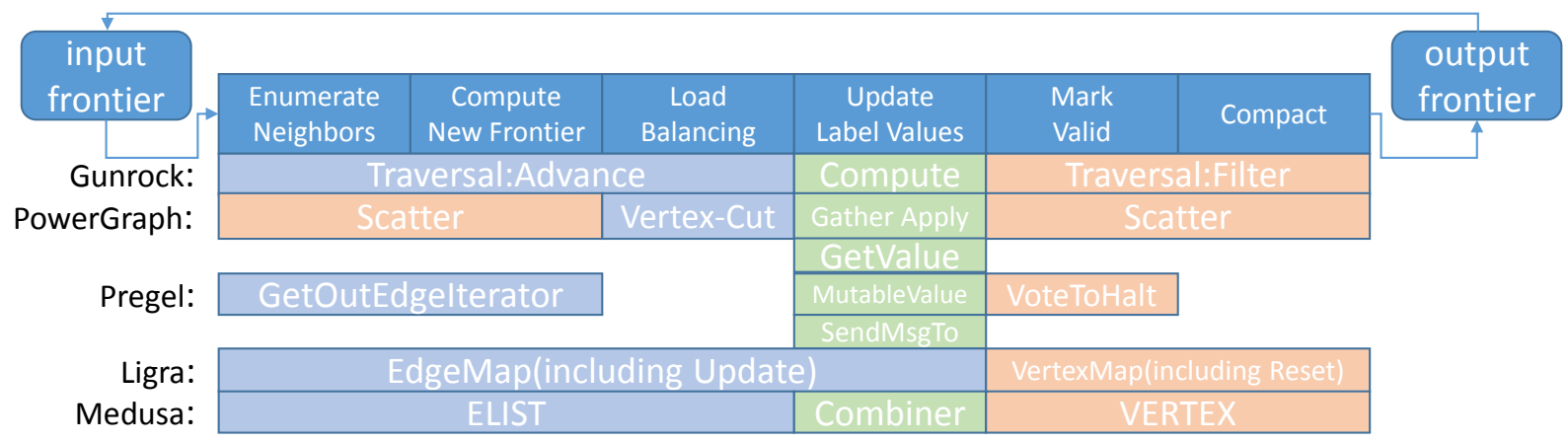

Figure 2: Operations that make up one iteration of SSSP and their mapping to the Gunrock, PowerGraph (GAS) [12], Pregel [20], Ligra [32], and Medusa [37] abstractions.

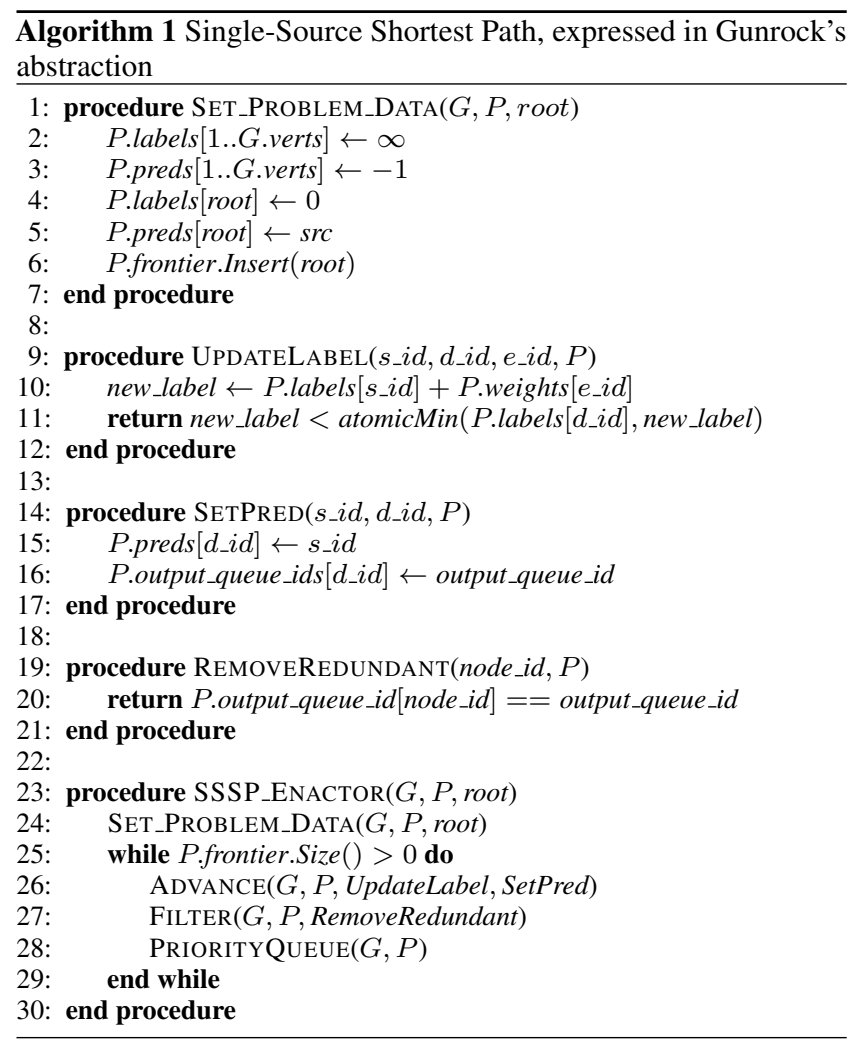

as a load balancing strategy, it replaces the large synchronization cost in edge-cut into a single-node synchronization cost. This is a productive strategy for multi-node implementations. GAS abstractions have successfully been mapped to the GPU, first with VertexAPI2 [7] and later with MapGraph [8] and CuSha [18]. GAS offers the twin benefits of simplicity and familiarity, given its popularity in the CPU world.

Recently, Wu et al. compared Gunrock vs. two GPU GAS frameworks, VertexAPI2 and MapGraph [36], demonstrating that Gunrock had appreciable performance advantages over the other two frameworks. One of the principal performance differences they identified comes from the significant fragmentation of GAS programs across many kernels that we discuss in more detail in Section 3.3. Applying automatic kernel fusion to GAS+GPU implementations could potentially help close their performance gap, but such an optimization is highly complex and has not yet appeared in any published work.
At a more fundamental level, we found that a compute-focused programming model like GAS was not flexible enough to manipulate the core frontier data structures in a way that enabled powerful features and optimizations such as push-pull and two-level priority queues; both fit naturally into Gunrock's abstraction. We believe bulk-synchronous operations on frontiers are a better fit than GAS for forward-looking GPU graph programming frameworks.

Message-passing Pregel [20] is a vertex-centric programming model that only provides data parallelism on vertices. For graphs with significant variance in vertex degree (e.g., power-law graphs), this would cause severe load imbalance on GPUs. The traversal operator in Pregel is general enough to apply to a wide range of graph primitives, but its vertex-centric design only achieves good parallelism when nodes in the graph have small and evenly-distributed neighborhoods. For real-world graphs that often have uneven distribution of node degrees, Pregel suffers from severe load imbalance. The Medusa GPU graph-processing framework [37] also implements a BSP model and allows computation on both edges and vertices. Medusa, unlike Gunrock, also allows edges and vertices to send messages to neighboring vertices. The Medusa authors note the complexity of managing the storage and buffering of these messages, and the difficulty of load-balancing when using segmented reduction for per-edge computation. Though they address both of these challenges in their work, the overhead of any management of messages is a significant contributor to runtime. Gunrock prefers the less costly direct communication between primitives and supports both push-based (scatter) communication and pull-based (gather) communication during traversal steps.

CPU strategies Ligra's powerful load-balancing strategy is based on CilkPlus, a fine-grained task-parallel library for CPUs. Despite promising GPU research efforts on task parallelism [4, 35], no such equivalent is available on GPUs, thus we implement our own loadbalancing strategies within Gunrock. Galois, like Gunrock, cleanly separates data structures from computation; their key abstractions are ordered and unordered set iterators that can add elements to sets during execution (such a dynamic data structure is a significant research challenge on GPUs). Galois also benefits from speculative parallel execution whose GPU implementation would also present a significant challenge. Both Ligra and Galois scale well within a node through inter-CPU shared memory; inter-GPU scalability, both due to higher latency and a lack of hardware support, is a much more manual, complex process.

Help's Primitives Help [29] characterizes graph primitives as a set of functions that enable special optimizations for different primitives at the cost of losing generality. Its Filter, Local Update of Vertices (LUV), Update Vertices Using One Other Vertex (UVUOV), and 
Aggregate Global Value (AGV) are all Gunrock filter operations with different computations. Aggregating Neighbor Values (ANV) maps to the advance operator in Gunrock. We also successfully implemented FS in Gunrock using two filter passes, one advance pass, and several other GPU computing primitives (sort, reduce, and scan).

Asynchronous execution Many CPU frameworks (e.g., Galois and GraphLab) efficiently incorporate asynchronous execution, but the GPU's expensive synchronization or locking operations would make this a poor choice for Gunrock. We do recover some of the benefits of prioritizing execution through our two-level priority queue.

\subsection{Gunrock's API and its Kernel-Fusion Optimization}

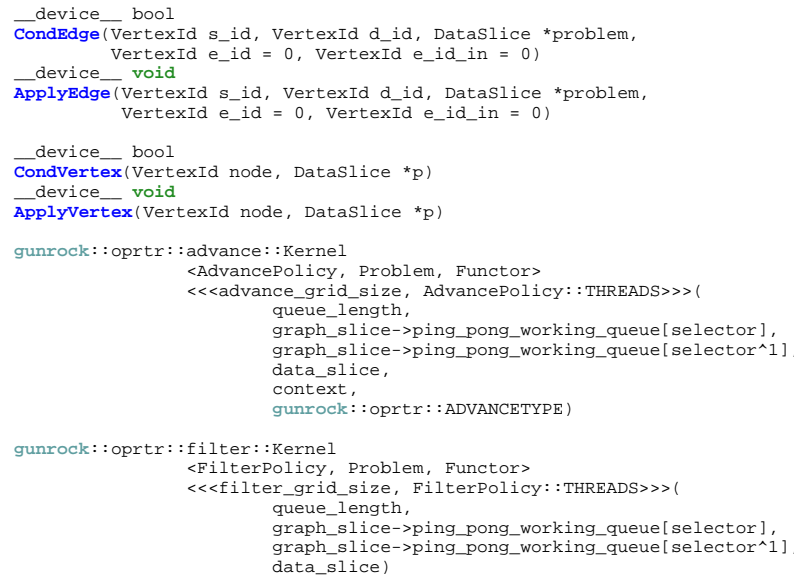

Figure 3: Gunrock's API set. Cond functors compute a boolean value per element, useful for filtering. Apply functors implement a compute operation on each element. User specific functor struct that contains its own implementation of these four functors is integrated at compile time into Advance or Filter kernels, providing automatic kernel fusion.

Gunrock programs specify three components: the problem, which provides graph topology data and an algorithm-specific data management interface; the functors, which contain user-defined computation code and expose kernel fusion opportunities that we discuss below; and an enactor, which serves as the entry point of the graph algorithm and specifies the computation as a series of advance and/or filter kernel calls with user-defined kernel launching settings.

Given Gunrock's abstraction, the most natural way to specify Gunrock programs would be as a sequence of bulk-synchronous steps, specified within the enactor and implemented as kernels, that operate on frontiers. Such an enactor is in fact the core of a Gunrock program, but an enactor-only program would sacrifice a significant performance opportunity. We analyzed the techniques that hardwired (primitive-specific) GPU graph primitives used to achieve high performance. One of their principal advantages is leveraging producer-consumer locality between operations by integrating multiple operations into single GPU kernels. Because adjacent kernels in CUDA or OpenCL share no state, combining multiple logical operations into a single kernel saves significant memory bandwidth that would otherwise be required to write and then read intermediate values to and from memory. The CUDA C++ programming environment we use has no ability to automatically fuse neighboring kernels together to achieve this efficiency (and automating this "kernel fusion" problem is a significant research challenge).

In particular, we noted that hardwired GPU implementations fuse regular computation steps together with more irregular steps like advance and filter by running a computation step (with regular parallelism) on the input or output of the irregularly-parallel step, all within the same kernel. To enable similar behavior in a programmable way, Gunrock exposes its computation steps as functors that are integrated into advance and filter kernels at compile time to achieve similar efficiency. We support functors that apply to \{edges, vertices $\}$ and either return a boolean value (the "cond" functor), useful for filtering, or perform a computation (the "apply" functor). These functors will then be integrated into "advance" and "filter" kernel calls, which hide any complexities of how those steps are internally implemented. We summarize the API for these operations in Figure 3. Our focus on kernel fusion enabled by our API design is absent from other programmable GPU graph libraries, but it is crucial for performance.

In terms of data structures, Gunrock represents all per-node and per-edge data as structure-of-array (SOA) data structures that allow coalesced memory accesses with minimal memory divergence. The data structure for the graph itself is perhaps even more important In Gunrock, we use a compressed sparse row (CSR) sparse matrix for vertex-centric operations by default and allow users to choose an edge-list-only representation for edge-centric operations. CSR uses a column-indices array, $C$, to store a list of neighbor vertices and a row-offsets array, $R$, to store the offset of the neighbor list for each vertex. It provides compact and efficient memory access, and allows us to use scan, a common and efficient parallel primitive, to reorganize sparse and uneven workloads into dense and uniform ones in all phases of graph processing [24].

We next provide detail on Gunrock's implementations of workload-mapping/load-balancing (Section 3.4) and optimizations (Section 3.5)

\subsection{Workload Mapping and Load Balancing Details}

Choosing the right abstraction is one key component in achieving high performance within a graph framework. The second component is optimized implementations of the primitives within the framework. One of Gunrock's major contributions is generalizing two workloaddistribution and load-balance strategies that each previously applied to a single hardwired GPU graph primitive into Gunrock's generalpurpose advance operator.

Gunrock's advance step generates an irregular workload. Consider an advance that generates a new vertex frontier from the neighbors of all vertices in the current frontier. If we parallelize over input vertices, graphs with a variation in vertex degree (with differentsized neighbor lists) will generate a corresponding imbalance in per-vertex work. Thus, mapping the workload of each vertex onto the GPU so that they can be processed in a load-balanced way is essential for efficiency.

The most significant previous work in this area balances load by cooperating between threads. Targeting BFS, Merrill et al. [24] map the workload of a single vertex to a thread, a warp, or a cooperative thread array (CTA), according to the size of its neighbor list. Targeting SSSP, Davidson et al. [5] use two load-balanced workload mapping strategies, one that groups input work and the other that groups output work. The first partitions the frontier into equally sized chunks and assigns all neighbor lists of one chunk to one block; the second partitions the neighbor list set into equally sized chunks (possibly splitting the neighbor list of one node into multiple chunks) and assigns each chunk of edge lists to one block of threads. Merrill et al. (unlike Davidson et al.) also supports the (BFSspecific) ability to process frontiers of edges rather than just frontiers of vertices. We integrate both techniques together, generalize them into a generic advance operator, and extend them by supporting an effective pull-based optimization strategy (Section 3.5). The result is the following two load-balancing strategies within Gunrock. 
Per-thread fine-grained One straightforward approach to load balancing is to map one frontier vertex's neighbor list to one thread. Each thread loads the neighbor list offset for its assigned node, then serially processes edges in its neighbor list. We have improved this method in several ways. First, we load all the neighbor list offsets into shared memory, then use a CTA of threads to cooperatively process per-edge operations on the neighbor list. Simultaneously, we use vertex-cut to split the neighbor list of a node so that it can be processed by multiple threads. We found out that this method performs better when used for large-diameter graphs with a relatively even degree distribution since it balances thread work within a CTA, but not across CTAs. For graphs with a more uneven degree distribution (e.g., scale-free social graphs), we turn to a second strategy.

Per-warp and per-CTA coarse-grained Significant differences in neighbor list size cause the worst performance with our per-thread fine-grained strategy. We directly address the variation in size by grouping neighbor lists into three categories based on their size, then individually processing each category with a strategy targeted directly at that size. Our three sizes are (1) lists larger than a CTA; (2) lists larger than a warp (32 threads) but smaller than a CTA; and (3) lists smaller than a warp. We begin by assigning a subset of the frontier to a block. Within that block, each thread owns one node. The threads that own nodes with large lists arbitrate for control of the entire block. All the threads in the block then cooperatively process the neighbor list of the winner's node. This procedure continues until all nodes with large lists have been processed. Next, all threads in each warp begin a similar procedure to process all the nodes whose neighbor lists are medium-sized lists. Finally, the remaining nodes are processed using our per-thread fine-grained workload-mapping strategy (Figure 4).

The specialization of this method allows higher throughput on frontiers with a high variance in degree distribution, but at the cost of higher overhead due to the sequential processing of the three different sizes.

Load-Balanced Partitioning Davidson et al. and Gunrock improve on this method by first organizing groups of edges into equal-length chunks and assigning each chunk to a block. This division requires us to find the starting and ending indices for all the blocks within the frontier. We use an efficient sorted search to map such indices with the scanned edge offset queue. When we start to process a neighbor list of a new node, we use binary search to find the node ID for the edges that are going to be processed. Using this method, we ensure load-balance both within a block and between blocks (Figure 5).

At the high level, Gunrock makes a load-balancing strategy decision depending on topology. We note that our coarse-grained (loadbalancing) traversal method performs better on social graphs with irregular distributed degrees, while the fine-grained method is superior on graphs where most nodes have small degrees. For this reason, in Gunrock we implement a hybrid of both methods on both vertex and edge frontiers, using the fine-grained dynamic grouping strategy for nodes with relatively smaller neighbor lists and the coarse-grained load-balancing strategy for nodes with relatively larger neighbor lists. Within the latter, we set a static threshold. When the frontier size is smaller than the threshold, we use coarsegrained load-balance over nodes, otherwise coarse-grained loadbalance over edges. We have found that setting this threshold to 4096 yields consistent high performance for tests across all Gunrockprovided graph primitives. Users can also change this value easily in the Enactor module for their own datasets or graph primitives. Superior load balancing is one of the most significant reasons why Gunrock outperforms other GPU frameworks [36].

\subsection{Gunrock's Optimizations}

One of our main goals in designing the Gunrock abstraction was to easily allow integrating existing and new alternatives and optimizations into our primitives to give more options to programmers. In general, we have found that our data-centric abstraction, and our focus on manipulating the frontier, has been an excellent fit for these alternatives and optimizations, compared to a more difficult implementation path for other GPU computation-focused abstractions. We offer three examples.

Idempotent vs. non-idempotent operations Because multiple elements in the frontier may share a common neighbor, an advance step may generate an output frontier that has duplicated elements. For some graph primitives (e.g., BFS) with "idempotent" operations, repeating a computation causes no harm, and Gunrock's filter step can perform a series of inexpensive heuristics to reduce, but not eliminate, redundant entries in the output frontier. Gunrock also supports a non-idempotent advance, which internally uses atomic operations to guarantee each element appears only once in the output frontier.

Push vs. pull traversal Other GPU programmable graph frameworks also support an advance step, of course, but because they are centered on vertex operations on an implicit frontier, they generally support only "push"-style advance: the current frontier of active vertices "pushes" active status to its neighbors to create the new frontier. Beamer et al. [1] described a "pull"-style advance on CPUs: instead of starting with a frontier of active vertices, pull starts with a frontier of unvisited vertices, generating the new frontier by filtering the unvisited frontier for vertices that have neighbors in the current frontier.

Beamer et al. showed this approach is beneficial when the number of unvisited vertices drops below the size of the current frontier. While vertex-centered GPU frameworks have found it challenging to integrate this optimization into their abstraction, our data-centric abstraction is a much more natural fit because we can easily perform more flexible operations on frontiers. Gunrock internally converts the current frontier into a bitmap of vertices, generates a new frontier of all unvisited nodes, then uses an advance step to "pull" the computation from these nodes' predecessors if they are valid in the bitmap.

With this optimization, we see a speedup on BFS of $1.52 \mathrm{x}$ for scalefree graphs and 1.28x for small-degree-large-diameter graphs. In an abstraction like Medusa, with its fixed method (segmented reduction) to construct frontiers, it would be a significant challenge to integrate a pull-based advance. Currently in Gunrock, this optimization is applied to BFS only, but in the future, more sophisticated BC and SSSP implementations could benefit from it as well.

Priority Queue A straightforward BSP implementation of an operation on a frontier treats each element in the frontier equally, i.e., with the same priority. Many graph primitives benefit from prioritizing certain elements for computation with the expectation that computing those elements first will save work overall (e.g., delta-stepping for SSSP [25]). Gunrock generalizes the approach of Davidson et al. [5] by allowing user-defined priority functions to organize an output frontier into "near" and "far" slices. This allows the GPU to use a simple and high-performance split operation to create and maintain the two slices. Gunrock then considers only the near slice in the next processing steps, adding any new elements that do not pass the near criterion into the far slice, until the near slice is exhausted. We then update the priority function and operate on the far slice.

Like other Gunrock steps, constructing a priority queue directly manipulates the frontier data structure. It is difficult to implement 


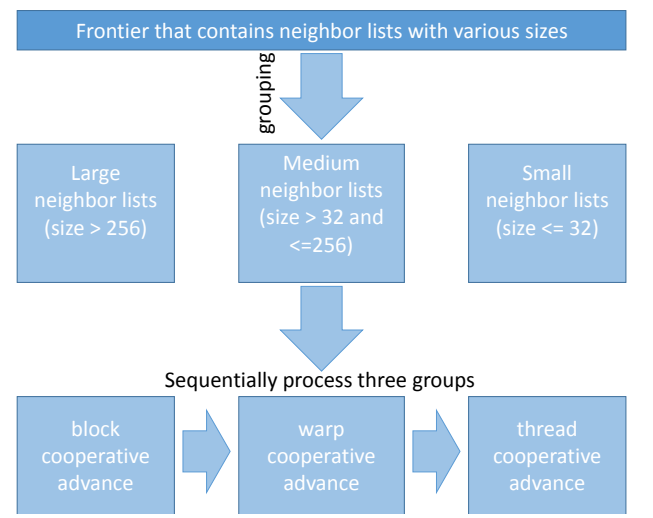

Figure 4: Load balancing strategy of Merrill et al. [24]

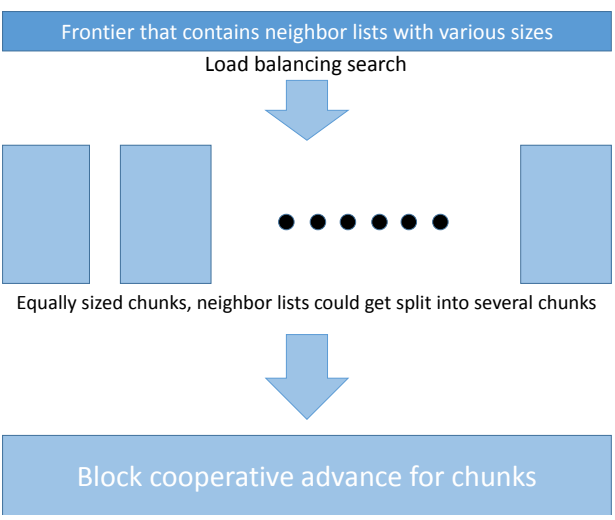

Figure 5: Load balancing strategy of Davidson et al. [5] such an operation in a GAS-based programming model since that programming model has no explicit way to reorganize a frontier.

Currently Gunrock uses this specific optimization only in SSSP, but we believe a workload reorganization strategy based on a more general priority queue implementation will enable a semiasynchronous execution model in Gunrock since different parts of frontier can process an arbitrary number of BSP steps. This will potentially increase the performance of various types of community detection and label propagation algorithms as well as algorithms on graphs with small "long tail" frontiers.

\section{Applications}

One of the principal advantages of Gunrock's abstraction is that our advance, filter, and compute steps can be composed to build new graph primitives with minimal extra work. For each primitive below, we describe the hardwired GPU implementation to which we compare, followed by how we express this primitive in Gunrock. Section 5 compares the performance between hardwired and Gunrock implementations.

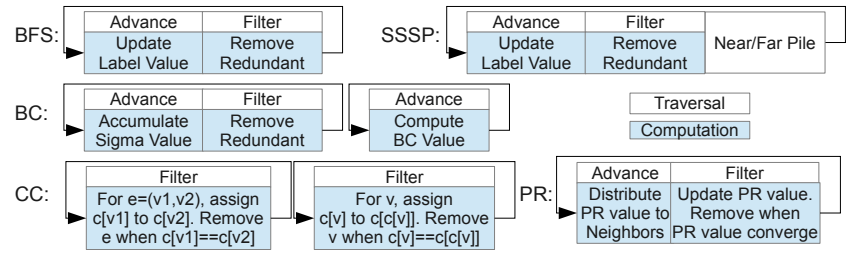

Figure 6: Operation flow chart for selected primitives in Gunrock (a black line with an arrow at one end indicates a while loop that runs until the frontier is empty).

\subsection{Breadth-First Search (BFS)}

BFS initializes its vertex frontier with a single source vertex. On each iteration, it generates a new frontier of vertices with all unvisited neighbor vertices in the current frontier, setting their depths and repeating until all vertices have been visited. BFS is one of the most fundamental graph primitives and serves as the basis of several other graph primitives.

Hardwired GPU Implementation The well-known BFS implementation of Merrill et al. [24] achieves its high performance through careful load-balancing, avoidance of atomics, and heuristics for avoiding redundant vertex discovery. Its chief operations are expand (to generate a new frontier) and contract (to remove redundant vertices) phases.
Gunrock Implementation Merrill et al.'s expand maps nicely to Gunrock's advance operator, and contract to Gunrock's filter operator. During advance, we set a label value for each vertex to show the distance from the source, and/or set a predecessor value for each vertex that shows the predecessor vertex's ID. We implement efficient load-balancing (Section 3.4) and both push- and pullbased advance (Section 3.5) for more efficient traversal. Our base implementation uses atomics during advance to prevent concurrent vertex discovery. When a vertex is uniquely discovered, we set its label (depth) and/or predecessor ID. Gunrock's fastest BFS uses the idempotent advance operator (thus avoiding the cost of atomics) and uses heuristics within its filter that reduce the concurrent discovery of child nodes (Section 3.5).

\subsection{Single-Source Shortest Path}

Single-source shortest path finds paths between a given source vertex and all other vertices in the graph such that the weights on the path between source and destination vertices are minimized. While the advance mode of SSSP is identical to BFS, the computation mode differs.

Hardwired GPU Implementation Currently the highest performing SSSP algorithm implementation on the GPU is the work from Davidson et al. [5]. They provide two key optimizations in their SSSP implementation: 1) a load balanced graph traversal method and 2) a priority queue implementation that reorganizes the workload. Gunrock generalizes both optimization strategies into its implementation, allowing them to apply to other graph primitives as well as SSSP. We implement Gunrock's priority queue as an additional filter pass between two iterations.

Gunrock Implementation We start from a single source vertex in the frontier. To compute a distance value from the source vertex, we need one advance and one filter operator. On each iteration, we visit all associated edges in parallel for each vertex in the frontier and relax the distance's value (if necessary) of the vertices attached to those edges. We use an AtomicMin to atomically find the minimal distance value we want to keep and a bitmap flag array associated with the frontier to remove redundant vertices. After each iteration, we use a priority queue to reorganize the vertices in the frontier.

\subsection{Betweenness Centrality}

The BC index can be used in social network analysis as an indicator of the relative importance of vertices in a graph. At a high level, the $\mathrm{BC}$ for a vertex in a graph is the fraction of shortest paths in a graph that pass through that vertex. Brandes's BC formulation [2] is most commonly used for GPU implementations. 
Hardwired GPU Implementation Brandes's formulation has two passes: a forward BFS pass to accumulate sigma values for each node, and a backward BFS pass to compute centrality values. Jia et al. [16] and Sariyüce et al. [31] both use an edge-parallel method to implement the above two passes. We achieve this in Gunrock using two advance operators on an edge frontier with different computations. The recent (hardwired) multi-GPU BC algorithm by McLaughlin and Bader [22] uses task parallelism, dynamic load balancing, and sampling techniques to perform BC computation in parallel from different sources on different GPU SMXs.

Gunrock Implementation Gunrock's implementation also contains two phases. The first phase has an advance step identical to the original BFS and a computation step that computes the number of shortest paths from source to each vertex. The second phase uses an advance step to iterate over the BFS frontier backwards with a computation step to compute the dependency scores. We achieve competitive performance on scale-free graphs with the latest hardwired BC algorithm [23]. Within Gunrock, we haven't yet considered task parallelism since it appears to be limited to BC, but it is an interesting area for future work.

\subsection{Connected Component Labeling}

The connected component primitive labels the vertices in each connected component in a graph with a unique component ID.

Hardwired GPU Implementation Soman et al. [34] base their implementation on two PRAM algorithms: hooking and pointerjumping. Hooking takes an edge as the input and tries to set the component IDs of the two end vertices of that edge to the same value. In odd-numbered iterations, the lower vertex writes its value to the higher vertex, and vice versa in the even numbered iteration. This strategy increases the rate of convergence. Pointer-jumping reduces a multi-level tree in the graph to a one-level tree (star). By repeating these two operators until no component ID changes for any node in the graph, the algorithm will compute the number of connected components for the graph and the connected component to which each node belongs.

Gunrock Implementation Gunrock uses a filter operator on an edge frontier to implement hooking. The frontier starts with all edges and during each iteration, one end vertex of each edge in the frontier tries to assign its component ID to the other vertex, and the filter step removes the edge whose two end vertices have the same component ID. We repeat hooking until no vertex's component ID changes and then proceed to pointer-jumping, where a filter operator on vertices assigns the component ID of each vertex to its parent's component ID until it reaches the root. Then a filter step removes the node whose component ID equals its own node ID. The pointerjumping phase also ends when no vertex's component ID changes.

\subsection{PageRank and Other Node Ranking Algorithms}

The PageRank link analysis algorithm assigns a numerical weighting to each element of a hyperlinked set of documents, such as the World Wide Web, with the purpose of quantifying its relative importance within the set. The iterative method of computing PageRank gives each vertex an initial PageRank value and updates it based on the PageRank of its neighbors, until the PageRank value for each vertex converges. PageRank is one of the simplest graph algorithms to implement on GPUs because the frontier always contains all vertices, so its computation is congruent to sparse matrix-vector multiply; because it is simple, most GPU frameworks implement it in a similar way and attain similar performance.

In Gunrock, we begin with a frontier that contains all vertices in the graph and end when all vertices have converged. Each iteration contains one advance operator to compute the PageRank value on the frontier of vertices, and one filter operator to remove the

\begin{tabular}{cccccc}
\hline Dataset & Vertices & Edges & Max Degree & Diameter & Type \\
\hline soc-orkut & $3 \mathrm{M}$ & $212.7 \mathrm{M}$ & 27,466 & 9 & $\mathrm{rs}$ \\
hollywood-09 & $1.1 \mathrm{M}$ & $112.8 \mathrm{M}$ & 11,467 & 11 & $\mathrm{rs}$ \\
indochina-04 & $7.4 \mathrm{M}$ & $302 \mathrm{M}$ & 256,425 & 26 & $\mathrm{rs}$ \\
kron_g500-logn21 & $2.1 \mathrm{M}$ & $182.1 \mathrm{M}$ & 213,904 & 6 & $\mathrm{gs}$ \\
rgg_n_24 & $16.8 \mathrm{M}$ & $265.1 \mathrm{M}$ & 40 & 2622 & $\mathrm{gm}$ \\
roadnet_CA & $2 \mathrm{M}$ & $5.5 \mathrm{M}$ & 12 & 849 & $\mathrm{rm}$ \\
\hline
\end{tabular}

Table 1: Dataset Description Table. Graph types are: r: real-world, g: generated, s: scale-free, and m: mesh-like.

vertices whose PageRanks have already converged. We accumulate PageRank values with AtomicAdd operations.

Bipartite graphs Geil et al. [9] used Gunrock to implement Twitter's who-to-follow algorithm ("Money" [11]), which incorporated three node-ranking algorithms based on bipartite graphs (Personalized PageRank, Stochastic Approach for Link-Structure Analysis (SALSA), and Hyperlink-Induced Topic Search (HITS)). Their implementation, the first to use a programmable framework for bipartite graphs, demonstrated that Gunrock's advance operator is flexible enough to encompass all three node-ranking algorithms, including a 2-hop traversal in a bipartite graph.

Beyond the five graph primitives we evaluate here, we have developed or are actively developing several other graph primitives in Gunrock, including minimal spanning tree, maximal independent set, graph coloring, Louvain's method for community detection, and graph matching.

\section{Experiments \& Results}

We ran all experiments in this paper on a Linux workstation with $2 \times 3.50 \mathrm{GHz}$ Intel 4-core, hyperthreaded E5-2637 v2 Xeon CPUs, $528 \mathrm{~GB}$ of main memory, and an NVIDIA K40c GPU with $12 \mathrm{~GB}$ on-board memory. GPU programs were compiled with NVIDIA's nvcc compiler (version 7.0.27) with the -O3 flag. The BGL and PowerGraph code were compiled using gec 4.8 .4 with the -O3 flag. Ligra was compiled using icpc 15.0.1 with CilkPlus. All results ignore transfer time (both disk-to-memory and CPU-to-GPU). All tests were run 10 times with the average runtime used for results.

The datasets used in our experiments are shown in Table 1. We converted all datasets to undirected graphs. The six datasets include both real-world and generated graphs; the topology of these datasets spans from regular to scale-free.

Soc-orkut (soc) and hollywood-09 (h09) are two social graphs; indochina-04 (i04) is a crawled hyperlink graph from indochina web domains; kron_g500-logn21 (kron) is a generated R-MAT graph. All four datasets are scale-free graphs with diameters of less than 20 and unevenly distributed node degrees ( $80 \%$ of nodes have degree less than 64).

Both rgg_n_24 (rgg) and roadnet_CA (roadnet) datasets have large diameters with small and evenly distributed node degrees (most nodes have degree less than 12).

soc is from Network Repository; i04, h09, and kron are from UF Sparse Matrix Collection; rgg is a random geometric graph we generated.

The edge weight values (used in SSSP) for each dataset are random values between 1 and 64 .

Performance Summary Tables 2 and 3, and Figure 7, compare Gunrock's performance against several other graph libraries and hardwired GPU implementations. In general, Gunrock's performance on BFS-based primitives (BFS, BC, and SSSP) shows comparatively better results when compared to other graph libraries on four scale-free graphs (soc, h09, i04, and kron), than on two 


\begin{tabular}{|c|c|c|c|c|c|c|c|c|c|c|c|}
\hline \multirow[b]{2}{*}{ Alg. } & \multirow[b]{2}{*}{ Dataset } & \multicolumn{5}{|c|}{ Runtime (ms) [lower is better] } & \multicolumn{5}{|c|}{ Edge throughput (MTEPS) [higher is better] } \\
\hline & & CuSha & MapGraph & $\begin{array}{l}\text { Hardwired } \\
\text { GPU }\end{array}$ & Ligra & Gunrock & CuSha & MapGraph & $\begin{array}{l}\text { Hardwired } \\
\text { GPU }\end{array}$ & Ligra & Gunrock \\
\hline \multirow{6}{*}{$\frac{n}{n}$} & soc & 251.8 & OOM & 45.43 & 27.2 & 47.23 & 844.7 & - & 4681 & 7819 & 4503 \\
\hline & h09 & 244.1 & 62.9 & 22.43 & 13.9 & 20.01 & 461.5 & 1791 & 5116 & 8100 & 5627 \\
\hline & i04 & 1809 & OOM & 84.08 & 223 & 62.12 & 164.8 & - & 4681 & 1337 & 4799 \\
\hline & kron & 237.9 & 162.7 & 37.33 & 18.5 & 19.15 & 765.2 & 1119 & 4877 & 9844 & 9510 \\
\hline & $\mathrm{rgg}$ & 52522 & OOM & 202.5 & 1020 & 351.4 & 5.048 & - & 1309 & 260 & 754.6 \\
\hline & roadnet & 288.5 & 61.66 & 8.21 & 82.1 & 31 & 19.14 & 89.54 & 672.9 & 67.25 & 178.1 \\
\hline \multirow{6}{*}{$\begin{array}{l}\hat{n} \\
\hat{n} \\
\hat{n}\end{array}$} & soc & - & OOM & $1106.6^{*}$ & 950 & 1088 & - & - & - & - & 195.5 \\
\hline & h09 & 1043 & OOM & $308.5 *$ & 281 & 100.4 & - & - & - & - & 1122 \\
\hline & i04 & - & OOM & OOM & 850 & 511.5 & - & - & - & - & 582.9 \\
\hline & kron & 315.5 & 540.8 & $677.7 *$ & 416 & 222.7 & - & - & - & - & 817.6 \\
\hline & rgg & - & OOM & OOM & 103000 & 117089 & - & - & - & - & 2.264 \\
\hline & roadnet & 1185 & 1285 & 224.2 & 451 & 222.1 & - & - & 24.63 & - & 24.86 \\
\hline \multirow{6}{*}{ 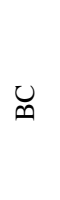 } & soc & - & - & 1044 & 223 & 721.2 & - & - & 407.4 & 1907 & 589.8 \\
\hline & h09 & - & - & 479.5 & 78.6 & 132.3 & - & - & 469.6 & 2867 & 1703 \\
\hline & i04 & - & - & 1389 & 557 & 164.3 & - & - & 429.1 & 1071 & 3630 \\
\hline & kron & - & - & 488.3 & 184 & 716.1 & - & - & 745.8 & 1979 & 508.5 \\
\hline & rgg & - & - & 25307 & 2720 & 1449 & - & - & 20.94 & 195 & 366 \\
\hline & roadnet & - & - & 256.8 & 232 & 120.6 & - & - & 42.99 & 47.6 & 91.57 \\
\hline \multirow{6}{*}{ 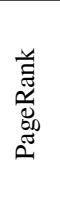 } & soc & 105.8 & OOM & - & 721 & 176 & & & & & \\
\hline & h09 & 43.27 & 94.35 & - & 107 & 27.31 & & & & & \\
\hline & i04 & 121.8 & OOM & - & 273 & 74.28 & & & & & \\
\hline & kron & 46.6 & 739.8 & - & 456 & 176.2 & & & & & \\
\hline & $\operatorname{rgg}$ & 48.6 & OOM & - & 307 & 80.42 & & & & & \\
\hline & roadnet & 0.864 & 8.069 & - & 14.6 & 6.691 & & & & & \\
\hline \multirow{6}{*}{ U } & soc & - & - & 91.58 & 313 & 252.9 & & & & & \\
\hline & h09 & - & - & 37.05 & 129 & 202.8 & & & & & \\
\hline & i04 & - & - & 120.8 & 535 & 2501 & & & & & \\
\hline & kron & - & - & 142.7 & 311 & 428.9 & & & & & \\
\hline & rgg & - & - & 109.6 & 3280 & 552.7 & & & & & \\
\hline & roadnet & - & - & 6.78 & 776 & 25.52 & & & & & \\
\hline
\end{tabular}

Table 3: Gunrock's performance comparison (runtime and edge throughput) with other graph libraries (CuSha, MapGraph, Ligra) and hardwired GPU implementations. SSSP MTEPS statistics are unavailable in most frameworks. All PageRank times are normalized to one iteration. Hardwired GPU implementations for each primitive are b40c (BFS) [24], delta-stepping SSSP [5] (numbers with * are achieved without delta-stepping optimization, otherwise will run out of memory), gpu_BC (BC) [31], and conn (CC) [34]. OOM means out-of-memory.

\begin{tabular}{ccccc}
\hline Algorithm & Galois & BGL & PowerGraph & Medusa \\
\hline BFS & 2.811 & - & - & 6.938 \\
SSSP & 0.725 & 52.04 & 6.207 & 11.88 \\
BC & 1.494 & - & - & - \\
PageRank & 1.94 & 337.6 & 9.683 & 8.982 \\
CC & 1.859 & 171.3 & 143.8 & - \\
\hline
\end{tabular}

Table 2: Geometric-mean runtime speedups of Gunrock on the datasets from Table 1 over frameworks not in Table 3. Due to Medusa's memory limitations, its SSSP and PageRank comparisons were measured on smaller datasets.

small-degree large-diameter graphs, rgg and roadnet. The primary reason is our load-balancing strategy during traversal (Table 4 shows Gunrock's superior performance on warp efficiency, a measure of load-balancing quality, across GPU frameworks and datasets), and particularly our emphasis on good performance for highly irregular graphs. As well, graphs with uniformly low degree expose less parallelism and would tend to show smaller gains in comparison to CPU-based methods.

vs. CPU Graph Libraries We compare Gunrock's performance with four CPU graph libraries: the Boost Graph Library (BGL) [33], one of the highest-performing CPU single-threaded graph li- braries [21]; PowerGraph, a popular distributed graph library [12]; and Ligra [32] and Galois [26, 28], two of the highest-performing multi-core shared-memory graph libraries. Against both BGL and PowerGraph, Gunrock achieves $6 x-337 x$ speedup on average on all primitives. Compared to Ligra, Gunrock's performance is generally comparable on most tested graph primitives; note Ligra uses both CPUs effectively. The performance inconsistency for SSSP vs. Ligra is due to comparing our Dijkstra-based method with Ligra's Bellman-Ford algorithm. Our SSSP's edge throughput is smaller than BFS but similar to BC because of similar computations (atomicMin vs. atomicAdd) and a larger number of iterations for convergence. The performance inconsistency for BC vs. Ligra on four scale-free graphs is because that Ligra applies pull-based traversal on BC while Gunrock has not yet done so. Compared to Galois, Gunrock shows more speedup on traversal-based graph primitives (BFS, SSSP, and BC) and less performance advantage on PageRank and $\mathrm{CC}$ due to their dense computation and more regular frontier structures.

vs. Hardwired GPU Implementations and GPU Libraries Compared to hardwired GPU implementations, depending on the dataset, Gunrock's performance is comparable or better on BFS, BC, and SSSP. For CC, Gunrock is 5x slower (geometric mean) than the hardwired GPU implementation due to irregular control flow because each iteration starts with full edge lists in both hooking and 


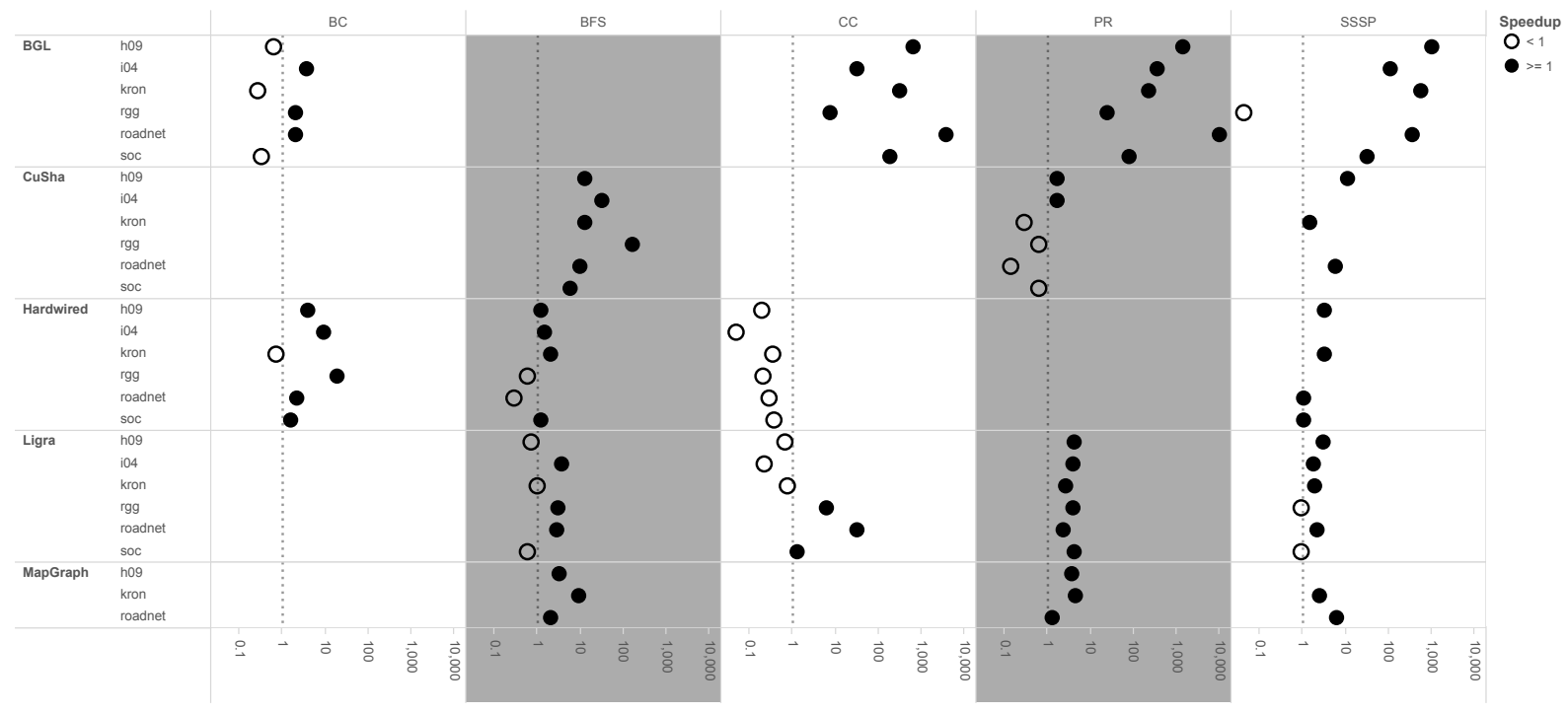

Figure 7: Execution-time speedup for Gunrock vs. five other graph processing libraries/hardwired algorithms on six different graph inputs. Data is from Table 3. Black dots indicate Gunrock is faster, white dots slower.

pointer-jumping phases. The alternative is extra steps to perform additional data reorganization. This tradeoff is not typical of our other primitives. While still achieving high performance, Gunrock's application code is smaller in size and clearer in logic compared to other GPU graph libraries ${ }^{1}$. Gunrock's Problem class (that defines problem data used for the graph algorithm) and kernel enactor are both template-based C++ code; Gunrock's functor code that specifies per-node or per-edge computation is C-like device code without any CUDA-specific keywords. For a new graph primitive, users only need to write from 133 (simple primitive, BFS) to 261 (complex primitive, SALSA) lines of code. Writing Gunrock code may require parallel programming concepts (e.g., atomics) but neither details of low-level GPU programming nor optimization knowledge.

Gunrock compares favorably to existing GPU graph libraries. MapGraph is faster than Medusa on all but one test [8] and Gunrock is faster than MapGraph on all tests: the geometric mean of Gunrock's speedups over MapGraph on BFS, SSSP, and PageRank are 4.3, 3.7, and 2.1, respectively. Gunrock also outperforms CuSha on BFS and SSSP. For PageRank, Gunrock achieves comparable performance without the G-Shard data preprocessing, which serves as the main load-balancing module in CuSha. The 1-GPU Gunrock implementation has $1.83 \mathrm{x}$ more MTEPS (4731 vs. 2590) on direction-optimized BFS on the soc-LiveJournal dataset (a smaller scale-free graph in their test set) than the 2-CPU, 2-GPU configuration of Totem [30] All three GPU BFS-based high-level-programming-model efforts (Medusa, MapGraph, and Gunrock) adopt load-balancing strategies from Merrill et al.'s BFS [24]. While we would thus expect Gunrock to show similar performance on BFS-based graph primitives as these other frameworks, we attribute our performance advantage to two reasons: (1) our improvements to efficient and load-balanced traversal that are integrated into the Gunrock core, and (2) a more powerful, GPU-specific programming model that allows more efficient high-level graph implementations. (1) is also the reason that Gunrock implementations can compete with hardwired implementa-

\footnotetext{
${ }^{1}$ We believe this assertion is true given our experience with other GPU libraries when preparing this evaluation section, but freely acknowledge this is nearly impossible to quantify. We invite readers to peruse our annotated code for BFS and SALSA at http://gunrock.github.io/gunrock/ doc/annotated_primitives/annotated_primitives.html.
}

\begin{tabular}{llcccccc}
\hline Alg. & Framework & soc & h09 & i04 & kron & rgg & roadnet \\
\hline \multirow{3}{*}{ BFS } & Gunrock & $97.39 \%$ & $97.35 \%$ & $97.97 \%$ & $97.73 \%$ & $96.72 \%$ & $97.01 \%$ \\
& MapGraph & - & $95.81 \%$ & - & $97.19 \%$ & - & $87.49 \%$ \\
& CuSha & $77.12 \%$ & $80.12 \%$ & $72.40 \%$ & $50.34 \%$ & $85.32 \%$ & $87.80 \%$ \\
\hline \multirow{2}{*}{ SSSP } & Gunrock & $83.35 \%$ & $82.56 \%$ & $83.18 \%$ & $85.15 \%$ & $82.84 \%$ & $83.47 \%$ \\
& MapGraph & - & - & - & $95.62 \%$ & - & $91.51 \%$ \\
& CuSha & $78.40 \%$ & $80.17 \%$ & $76.63 \%$ & $52.72 \%$ & $86.96 \%$ & $85.28 \%$ \\
\hline \multirow{2}{*}{ PR } & Gunrock & $99.56 \%$ & $99.42 \%$ & $99.54 \%$ & $99.43 \%$ & $99.52 \%$ & $99.49 \%$ \\
& MapGraph & - & $98.97 \%$ & - & $99.16 \%$ & - & $96.27 \%$ \\
& CuSha & $82.29 \%$ & $87.26 \%$ & $85.10 \%$ & $63.46 \%$ & $91.04 \%$ & $89.23 \%$ \\
\hline
\end{tabular}

Table 4: Average warp execution efficiency (fraction of threads active during computation). This figure is a good metric for the quality of a framework's load-balancing capability. (- indicates the graph framework ran out of memory.)

tions; we believe Gunrock's load-balancing and work distribution strategies are at least as good as if not better than the hardwired primitives we compare against. Gunrock's memory footprint is at the same level as Medusa and better than MapGraph (note the OOM test cases for MapGraph in Table 3). The data size is $\alpha|E|+\beta|V|$ for current graph primitives, where $|E|$ is the number of edges, $|V|$ is the number of nodes, and $\alpha$ and $\beta$ are both integers where $\alpha$ is usually 1 and at most 3 (for BC) and $\beta$ is between 2 to 8 .

Figure 8 shows how different optimization strategies improve the performance of graph traversal; here we use BFS as an example. As noted in Section 3.4, the load-balancing traversal method works better on social graphs with irregular distributed degrees, while the Thread-Warp-CTA method works better on graphs where most nodes have small degrees. The direction-optimal traversal strategy also works better on social graphs, whereas on the road-network and bitcoin-transactions graph, we see less concurrent discovery and the performance benefits are not as significant. In general, we can predict which strategies will be most beneficial based only on the degree distribution; many application scenarios may allow precomputation of this distribution and thus we can choose the optimal strategies before we begin computation. 

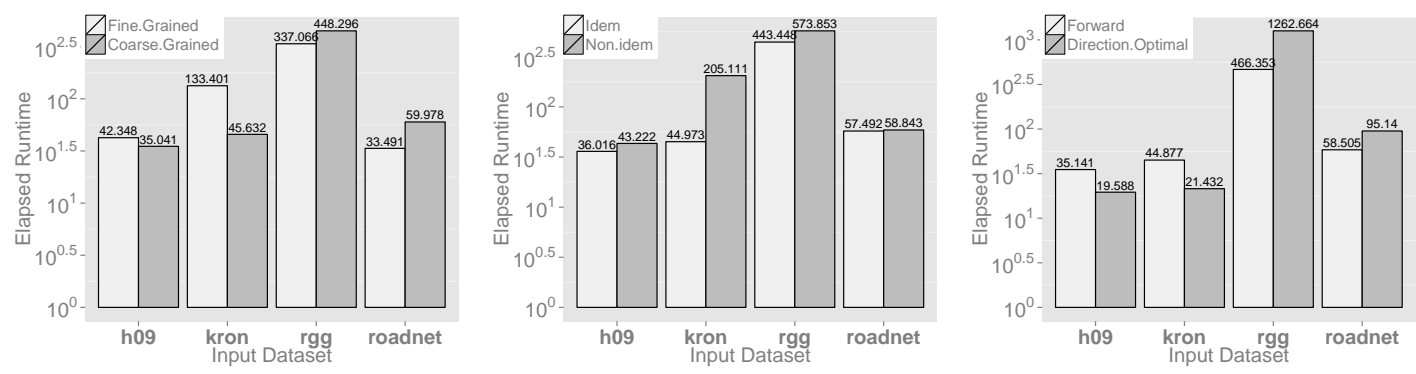

Figure 8: Left: Performance comparison with two different workload mapping optimizations. Middle: Performance comparison on graph traversal with idempotent operations enabled vs. disabled. Right: Performance comparison between forward and direction optimal graph traversal.

\section{Future Work}

We believe Gunrock currently provides an excellent foundation for developing GPU-based graph primitives. We hope to extend Gunrock with the following improvements:

Dynamic graphs While Gunrock currently implements several graph primitives (e.g., minimum spanning tree and connected components) that internally modify graph topology, generalized support of dynamic graphs on GPUs that change their structure during computation is still an unsolved problem.

Global, neighborhood, and sampling operations In Gunrock, computation on vertices and edges is convenient and fast. However, global and neighborhood operations, such as reductions over neighbor lists, generally require less-efficient atomic operations and are an ongoing challenge. We believe a new gather-reduce operator on neighborhoods associated with vertices in the current frontier both fits nicely into Gunrock's abstraction and will significantly improve performance on this operation. We also expect to explore a "sample" step that can take a random subsample of a frontier, which we can use to compute a rough or seeded solution that may allow faster convergence on a full graph.

Kernel fusion Gunrock's implementation generally allows more opportunities to fuse multiple operations into a single kernel than GAS+GPU implementations (Section 3.3), but does not achieve the level of fusion of hardwired implementations. This interesting (and unsolved, in the general case) research problem represents the largest performance gap between hardwired and Gunrock primitives.

Scalability Today, Gunrock is implemented for single-GPU computation and graphs that fit into the GPU's memory. We believe the contributions in this paper successfully advance the state of the art on one GPU, but for greater impact, a future Gunrock must scale in three directions: to leverage the larger memory capacity of a CPU host; to multiple GPUs on a single node; and to a distributed, multi-node clustered system. Current GPU work in this area generally targets only specific primitives (e.g., Merrill et al.'s. multi-GPU BFS [24]) and/or is not performance-competitive with large-memory, single-node CPU implementations. We hope that Gunrock's data-centric focus on frontiers-which we believe is vital for data distributions that go beyond a single GPU's memoryprovides an excellent substrate for a future scalable GPU-based graph implementation.

\section{Conclusions}

Gunrock was born when we spent two months writing a single hardwired GPU graph primitive. We knew that for GPUs to make an impact in graph analytics, we had to raise the level of abstraction in building graph primitives. From the beginning, we designed
Gunrock with the GPU in mind, and its data-centric, frontier-focused abstraction has proven to map naturally to the GPU, giving us both good performance and good flexibility. We have also found that implementing this abstraction has allowed us to integrate numerous optimization strategies, including multiple load-balancing strategies for traversal, direction-optimal traversal, and a two-level priority queue. The result is a framework that is general (able to implement numerous simple and complex graph primitives), straightforward to program (new primitives only take a few hundred lines of code and require minimal GPU programming knowledge), and fast (on par with hardwired primitives and faster than any other programmable GPU graph library).

\section{Acknowledgments}

We thank Joe Mako for providing the speedup chart design. Also, thanks to the DARPA XDATA program and our program managers Christopher White and Wade Shen (US Army award W911QX-12C-0059); DARPA STTR awards D14PC00023 and D15PC00010; NSF awards CCF-1017399 and OCI-1032859; and UC Lab Fees Research Program Award 12-LR-238449.

\section{References}

[1] S. Beamer, K. Asanović, and D. Patterson. Direction-optimizing breadth-first search. In Proceedings of the International Conference on High Performance Computing, Networking, Storage and Analysis, SC '12, pages 12:1-12:10, Nov. 2012.

[2] U. Brandes. A faster algorithm for betweenness centrality. Journal of Mathematical Sociology, 25(2):163-177, 2001.

[3] M. Burtscher, R. Nasre, and K. Pingali. A quantitative study of irregular programs on GPUs. In IEEE International Symposium on Workload Characterization, IISWC-2012, pages 141-151, Nov. 2012.

[4] D. Cederman and P. Tsigas. On dynamic load-balancing on graphics processors. In Graphics Hardware 2008, pages 57-64, June 2008.

[5] A. Davidson, S. Baxter, M. Garland, and J. D. Owens. Work-efficient parallel GPU methods for single source shortest paths. In Proceedings of the 28th IEEE International Parallel and Distributed Processing Symposium, pages 349-359, May 2014.

[6] D. Delling, A. V. Goldberg, A. Nowatzyk, and R. F. Werneck. PHAST: Hardware-accelerated shortest path trees. Journal of Parallel and Distributed Computing, 73:940-952, Sept. 2010.

[7] E. Elsen and V. Vaidyanathan. A vertex-centric CUDA/C++ API for large graph analytics on GPUs using the gather-apply-scatter abstraction, 2013. http://www.github.com/RoyalCaliber/ vertexAPI2.

[8] Z. Fu, M. Personick, and B. Thompson. MapGraph: A high level API for fast development of high performance graph analytics on GPUs. In Proceedings of the Workshop on GRAph Data Management Experiences and Systems, GRADES ’14, pages 2:1-2:6, June 2014. 
[9] A. Geil, Y. Wang, and J. D. Owens. WTF, GPU! Computing Twitter's who-to-follow on the GPU. In Proceedings of the Second ACM Conference on Online Social Networks, COSN '14, Oct. 2014.

[10] R. Geisberger, P. Sanders, and D. Schultes. Better approximation of betweenness centrality. In Proceedings of the Tenth Workshop on Algorithm Engineering and Experiments, ALENEX08, pages 90-100, Jan. 2008.

[11] A. Goel. The "who-to-follow" system at Twitter: Algorithms, impact, and further research. WWW 2014 industry track, 2014.

[12] J. E. Gonzalez, Y. Low, H. Gu, D. Bickson, and C. Guestrin. PowerGraph: Distributed graph-parallel computation on natural graphs. In Proceedings of the 10th USENIX Conference on Operating Systems Design and Implementation, OSDI '12, pages 17-30. USENIX Association, Oct. 2012.

[13] D. Gregor and A. Lumsdaine. The parallel BGL: A generic library for distributed graph computations. In Parallel Object-Oriented Scientific Computing (POOSC), July 2005.

[14] J. Greiner. A comparison of parallel algorithms for connected components. In Proceedings of the Sixth Annual ACM Symposium on Parallel Algorithms and Architectures, SPAA '94, pages 16-25, June 1994.

[15] S. Hong, H. Chafi, E. Sedlar, and K. Olukotun. Green-Marl: A DSL for easy and efficient graph analysis. In Proceedings of the Seventeenth International Conference on Architectural Support for Programming Languages and Operating Systems, ASPLOS XVII, pages 349-362, Mar. 2012.

[16] Y. Jia, V. Lu, J. Hoberock, M. Garland, and J. C. Hart. Edge v. node parallelism for graph centrality metrics. In W. W. Hwu, editor, GPU Computing Gems Jade Edition, chapter 2, pages 15-28. Morgan Kaufmann, Oct. 2011.

[17] S. W. Keckler, W. J. Dally, B. Khailany, M. Garland, and D. Glasco. GPUs and the future of parallel computing. IEEE Micro, 31(5):7-17, Sept. 2011.

[18] F. Khorasani, K. Vora, R. Gupta, and L. N. Bhuyan. CuSha: Vertexcentric graph processing on GPUs. In Proceedings of the 23rd International Symposium on High-performance Parallel and Distributed Computing, HPDC'14, pages 239-252, June 2014.

[19] Y. Low, J. Gonzalez, A. Kyrola, D. Bickson, C. Guestrin, and J. M. Hellerstein. GraphLab: A new parallel framework for machine learning. In Proceedings of the Twenty-Sixth Annual Conference on Uncertainty in Artificial Intelligence, UAI-10, pages 340-349, July 2010.

[20] G. Malewicz, M. H. Austern, A. J. C. Bik, J. C. Dehnert, I. Horn, N. Leiser, and G. Czajkowski. Pregel: A system for large-scale graph processing. In Proceedings of the 2010 ACM SIGMOD International Conference on Management of Data, SIGMOD '10, pages 135-146, June 2010.

[21] R. C. McColl, D. Ediger, J. Poovey, D. Campbell, and D. A. Bader A performance evaluation of open source graph databases. In Proceedings of the First Workshop on Parallel Programming for Analytics Applications, PPAA '14, pages 11-18, Feb. 2014.

[22] A. McLaughlin and D. A. Bader. Scalable and high performance betweenness centrality on the GPU. In Proceedings of the International Conference for High Performance Computing, Networking, Storage and Analysis, SC14, pages 572-583, Nov. 2014.

[23] A. McLaughlin, J. Riedy, and D. A. Bader. A fast, energy-efficient abstraction for simultaneous breadth-first searches. In 2015 IEEE High Performance Extreme Computing Conference, HPEC '15, Sept. 2015.

[24] D. Merrill, M. Garland, and A. Grimshaw. Scalable GPU graph traversal. In Proceedings of the 17th ACM SIGPLAN Symposium on Principles and Practice of Parallel Programming, PPoPP'12, pages 117-128, Feb. 2012.

[25] U. Meyer and P. Sanders. $\Delta$-stepping: a parallelizable shortest path algorithm. Journal of Algorithms, 49(1):114-152, Oct. 2003. 1998 European Symposium on Algorithms.

[26] D. Nguyen, A. Lenharth, and K. Pingali. A lightweight infrastructure for graph analytics. In Proceedings of ACM Symposium on Operating Systems Principles, SOSP' 13, pages 456-471, Nov. 2013.
[27] P. R. Pande and D. A. Bader. Computing betweenness centrality for small world networks on a GPU. In HPEC, 2011.

[28] K. Pingali, D. Nguyen, M. Kulkarni, M. Burtscher, M. A. Hassaan, R. Kaleem, T.-H. Lee, A. Lenharth, R. Manevich, M. Méndez-Lojo, D. Prountzos, and X. Sui. The tao of parallelism in algorithms. In Proceedings of the 32nd ACM SIGPLAN Conference on Programming Language Design and Implementation, PLDI '11, pages 12-25, June 2011.

[29] S. Salihoglu and J. Widom. HelP: High-level primitives for large-scale graph processing. In Proceedings of the Workshop on GRAph Data Management Experiences and Systems, GRADES '14, pages 3:1-3:6, June 2014.

[30] S. Sallinen, A. Gharaibeh, and M. Ripeanu. Accelerating directionoptimized breadth first search on hybrid architectures. CoRR, abs/1503.04359(1503.04359v1), Mar. 2015.

[31] A. E. Sariyüce, K. Kaya, E. Saule, and U. V. Çatalyürek. Betweenness centrality on GPUs and heterogeneous architectures. In Proceedings of the 6th Workshop on General Purpose Processor Using Graphics Processing Units, GPGPU-6, pages 76-85, Mar. 2013.

[32] J. Shun and G. E. Blelloch. Ligra: a lightweight graph processing framework for shared memory. In Proceedings of the 18th ACM SIGPLAN Symposium on Principles and Practice of Parallel Programming, PPoPP' 13, pages 135-146, Feb. 2013.

[33] J. G. Siek, L.-Q. Lee, and A. Lumsdaine. The Boost Graph Library: User Guide and Reference Manual. Addison-Wesley, Dec. 2001.

[34] J. Soman, K. Kishore, and P. J. Narayanan. A fast GPU algorithm for graph connectivity. In 24th IEEE International Symposium on Parallel and Distributed Processing, Workshops and PhD Forum, IPDPSW 2010, pages 1-8, Apr. 2010.

[35] S. Tzeng, B. Lloyd, and J. D. Owens. A GPU task-parallel model with dependency resolution. IEEE Computer, 45(8):34-41, Aug. 2012.

[36] Y. Wu, Y. Wang, Y. Pan, C. Yang, and J. D. Owens. Performance characterization for high-level programming models for GPU graph analytics. In IEEE International Symposium on Workload Characterization, IISWC-2015, pages 66-75, Oct. 2015.

[37] J. Zhong and B. He. Medusa: Simplified graph processing on GPUs. IEEE Transactions on Parallel and Distributed Systems, 25(6):15431552, June 2014. 